\title{
Controllability and Observability of Nonautonomous Riesz-Spectral Systems
}

\author{
Sutrima Sutrima $\left(\mathbb{D},{ }^{1,2}\right.$ Christiana Rini Indrati, ${ }^{2}$ and Lina Aryati ${ }^{2}$ \\ ${ }^{1}$ Mathematics Department, Universitas Sebelas Maret, Surakarta, Indonesia \\ ${ }^{2}$ Mathematics Department, Universitas Gadjah Mada, Yogyakarta, Indonesia \\ Correspondence should be addressed to Sutrima Sutrima; zutrima@yahoo.co.id
}

Received 8 February 2018; Accepted 5 April 2018; Published 15 May 2018

Academic Editor: Khalil Ezzinbi

Copyright (c) 2018 Sutrima Sutrima et al. This is an open access article distributed under the Creative Commons Attribution License, which permits unrestricted use, distribution, and reproduction in any medium, provided the original work is properly cited.

\begin{abstract}
There are many industrial and biological reaction diffusion systems which involve the time-varying features where certain parameters of the system change during the process. A part of the transport-reaction phenomena is often modelled as an abstract nonautonomous equation generated by a (generalized) Riesz-spectral operator on a Hilbert space. The basic problems related to the equations are existence of solutions of the equations and how to control dynamical behaviour of the equations. In contrast to the autonomous control problems, theory of controllability and observability for the nonautonomous systems is less well established. In this paper, we consider some relevant aspects regarding the controllability and observability for the nonautonomous Riesz-spectral systems including the Sturm-Liouville systems using a $C_{0}$-quasi-semigroup approach. Three examples are provided. The first is related to sufficient conditions for the existence of solutions and the others are to confirm the approximate controllability and observability of the nonautonomous Riesz-spectral systems and Sturm-Liouville systems, respectively.
\end{abstract}

\section{Introduction}

In the real problems, many underlying transport-reaction phenomena are described by partial differential equations with the time-varying coefficients. The phenomena arise in processes such as crystal growth, metal casting and annealing, solid-gas reaction systems (see [1-3]), and heat conduction of a material undergoing decay or radioactive damage [4]. The others also arise in solid-fluid mechanics and biological systems. The time-dependencies of the system parameters can be caused by changes in the boundary of domain and variances in the diffusion characteristics. The transport-reaction phenomena encourage the emergence of nonautonomous linear control systems.

Let $X, U$, and $Y$ be complex Hilbert spaces. Suppose that $B(t): U \rightarrow X$ and $C(t): X \rightarrow Y$ are bounded operators such that $B(\cdot) \in L_{\infty}\left(\mathbb{R}^{+}, \mathscr{L}_{s}(U, X)\right)$ and $C(\cdot) \in L_{\infty}\left(\mathbb{R}^{+}, \mathscr{L}_{s}(X, Y)\right)$, where $\mathscr{L}_{s}(V, W)$ denotes the space of bounded operators from $V$ to $W$ equipped with strong operator topology. We consider the linear nonautonomous control systems on $X$ with state $x$, input $u$, and output $y$ :

$$
\begin{aligned}
& \dot{x}(t)=A(t) x(t)+B(t) u(t), \quad t \geq 0, x(0)=x_{0}, \\
& y(t)=C(t) x(t)
\end{aligned}
$$

where $x$ is an unknown function from real interval $[0, \infty)$ into $X$ and $A(t)$ is a linear closed operator in $X$ with domain $\mathscr{D}(A(t))=\mathscr{D}$, independent of $t$ and dense in $X$. We denote the state linear system (1)-(2) by $(A(t), B(t), C(t))$. To avoid clutter, we also use notations $(A(t), B(t),-)$ and $(A(t),-, C(t))$ if $C(t)=0$ and $B(t)=0$, respectively.

There is an extensive amount of literatures which have studied controllability for the system $(A(t), B(t),-)$ (1). Barcenas and Leiva [5] prove some properties of attainable sets for the systems (1) with time-varying constrained controls and target sets. They also characterize the extremal controls and give necessary and sufficient conditions for the normality of the system. Elharfi et al. [6] study wellposedness of a class of nonautonomous neutral control systems in Banach spaces. The systems are represented by absolutely regular nonautonomous linear systems in the sense of Schnaubelt [7]. These works can be considered as 
the nonautonomous version of the works of Bounit and Hadd [8]. By employing skew-product semiflow technique, Barcenas et al. [9] give necessary and sufficient conditions for exact and approximate controllability of a wide class of linear infinite-dimensional nonautonomous control systems (1). $\mathrm{Ng}$ et al. [10] characterize the some pertinent aspects regarding the controllability and observability of system (1)-(2) which are modelled by parabolic partial differential equations with time-varying coefficients. By using theory of linear evolution system and Schauder fixed point theorem, Fu and Zhang [11] establish a sufficient result of exact null controllability for a nonautonomous functional evolution system with nonlocal conditions. Using evolution operators and concept of Lebesgue extensions, Hadd [12] proposes a new approach which brings nonautonomous linear systems with state, input, and output delays in line with the standard theory. Leiva and Barcenas [13] have established a quasisemigroup theory as an alternative approach in solving (1). Even the control theory can be developed by this approach although it is still limited to the time-invariant controls [14]. In this context, $A(t)$ is an infinitesimal generator of a $C_{0}$-quasi-semigroup on $X$. Finally, the advanced properties and some types of stabilities of the $\mathrm{C}_{0}$-quasi-semigroups in Banach spaces can be determined by Sutrima et al. [15] and Sutrima et al. [16], respectively. These results are important in analysis and applications of the $C_{0}$-quasi-semigroups.

In the autonomous case, that is, $A(t)=A, B(t)=B$, and $C(t)=C$, independent of $t$, there are many literatures which have been devoted to study of the controllability and observability for the system $(A, B, C)$ of (1)-(2). Dolecki and Russell [17] explore the duality relationships between observation and control in an abstract Banach space setting. Investigation is also given to the problem of optimal reconstruction of system states from observations. Zhao and Weiss [18] establish the well-posedness, regularity, exact (approximate) controllability, and exact (approximate) observability results for the coupled systems consisting of a well-posed and regular subsystem and a finite-dimensional subsystem connected in feedback. For neutral type linear systems in Hilbert spaces, Rabah et al. [19] prove that exact null controllability and complete stabilizability are equivalent. The paper also considers the case when the feedback is not bounded. In particular, if $A$ is a Riesz-spectral generator of a $C_{0}$-semigroup on $X$, then the solution of (1) for $B=0$ can be expressed as an infinite sum of all its eigenvectors which form a Riesz basis (see $[20,21])$, and in this case the system $(A, B,-)$ is called a Riesz-spectral system. It gives convenience to analyze some problems in infinite-dimensional systems such as spectrumdetermined growth condition, controllability, observability, stabilizability, and detectability; see, for example, [22, 23].

Although the aforementioned researches provide a wellestablished theoretical basis on the nonautonomous Cauchy problems and the controllability and observability theory, there are a relatively scarce number of the researches using quasi-semigroups. Even, there is no research which investigates the Riesz-spectral systems on Hilbert space for the nonautonomous cases. These are challenges to study and to realize the associated control problems, the controllability, and observability, for the nonautonomous infinite-dimensional systems.

In this paper, we are concerned with investigation of sufficient conditions for $A(t)$ to induce a nonautonomous Rieszspectral system. The obtained nonautonomous operator is implemented to study the controllability and observability for the nonautonomous systems. All the studies use the $C_{0^{-}}$ quasi-semigroup approach. The organization of this paper is as follows. In Section 2, we provide notion of the generalized Riesz-spectral operator and its sufficiency related to the nonautonomous systems. The concepts of controllability and observability for the nonautonomous systems are considered in the Section 3. In Section 4, we confirm the obtained results by the two examples.

\section{Generalized Riesz-Spectral Generator}

This section is a part of the main results. We first recall the definition of a strongly continuous quasi-semigroups following $[13,14]$.

Definition 1. Let $\mathscr{L}(X)$ be the set of all bounded linear operators on Hilbert space $X$. A two-parameter commutative family $\{R(t, s)\}_{s, t \geq 0}$ in $\mathscr{L}(X)$ is called a strongly continuous quasi-semigroup, in short $C_{0}$-quasi-semigroup, on $X$ if, for each $r, s, t \geq 0$ and $x \in X$,

(a) $R(t, 0)=I$, identity operator on $X$,

(b) $R(t, s+r)=R(t+r, s) R(t, r)$,

(c) $\lim _{s \rightarrow 0^{+}}\|R(t, s) x-x\|=0$,

(d) there exists a continuous increasing function $M$ : $[0, \infty) \rightarrow[0, \infty)$ such that

$$
\|R(t, s)\| \leq M(s) .
$$

In the sequel, for simplicity we denote the quasisemigroup $\{R(t, s)\}_{s, t \geq 0}$ and family $\{A(t)\}_{t \geq 0}$ by $R(t, s)$ and $A(t)$, respectively.

In this section we investigate sufficient conditions of $A(t)$ such that (1) forms a nonautonomous Riesz-spectral system. It is well known that if $A$ is a Riesz-spectral operator, then it can be represented as an infinite sum of all its eigenvectors. However, as declared in Section 1 for nonautonomous system (1), we assume that $\mathscr{D}=\mathscr{D}(A(t))$ is independent of $t$. This implies that to be a Riesz-spectral operator, $A(t)$ has to have eigenvectors which are independent of $t$. A class that meets this criterion is a family of operators whose representation is as follows:

$$
A(t)=a(t) A,
$$

where $A$ is a Riesz-spectral operator on $X$ and $a$ is a bounded continuous function such that $a(t)>0, t \geq 0$. It is clear that, for every $t \geq 0, A(t)$ and $A$ have the common domain and eigenvectors. Moreover, if $\lambda_{n}, n \in \mathbb{N}$, is an eigenvalue of $A$, then $a(t) \lambda_{n}$ are the eigenvalues of $A(t)$ of (4). Hence, in general $A(t)$ may have the nonsimple eigenvalues. In case $A(t)$ is a differential operator, then the operator $A(t, \xi)$ of [10] satisfying the conditions $\mathrm{P} 1$ and $\mathrm{P} 2$ verifies (4). These urge the following notion. 
Definition 2. For every $t \geq 0$, let $A(t)$ be an operator of form (4) on a Hilbert space $X . A(t)$ is called a generalized Rieszspectral operator if $A$ is a Riesz-spectral operator.

Definition 2 states that if $a$ is a nonnegative constant function, then $A(t)$ is a Riesz-spectral operator. In the sequel we always assume that, for every $t \geq 0, A(t)$ is an operator of form (4). The following results are generalization of the results of $[21,22]$ for autonomous case.

Theorem 3. For every $t \geq 0$, let $A(t)$ be an operator of (4) where $A$ is a Riesz-spectral operator with simple eigenvalues $\left\{\lambda_{n}: n \in \mathbb{N}\right\}$ and corresponding eigenvectors $\left\{\phi_{n}: n \in \mathbb{N}\right\}$. If $\left\{\varphi_{n}: n \in \mathbb{N}\right\}$ are the eigenvectors of $A^{*}$, the adjoint of $A$, such that $\left\langle\phi_{n}, \varphi_{m}\right\rangle=\delta_{m n}$, then

(a) $\rho(A(t))=\{\lambda a(t): \lambda \in \rho(A)\}, \sigma(A(t))=\{\lambda a(t)$ : $\lambda \in \sigma(A)\}$, and for $\lambda \in \rho(A(t))$, the resolvent operator $\mathscr{R}(\lambda, A(t))$ is given by

$$
\mathscr{R}(\lambda, A(t)) x=\sum_{n=1}^{\infty} \frac{1}{\lambda-a(t) \lambda_{n}}\left\langle x, \varphi_{n}\right\rangle \phi_{n} ;
$$

(b) $A(t)$ has representation

$$
A(t) x=a(t) \sum_{n=1}^{\infty} \lambda_{n}\left\langle x, \varphi_{n}\right\rangle \phi_{n}
$$

for $x \in \mathscr{D}=\mathscr{D}(A(t))$, where

$$
\mathscr{D}=\left\{x \in X: \sum_{n=1}^{\infty}\left|\lambda_{n}\right|^{2}\left|\left\langle x, \varphi_{n}\right\rangle\right|^{2}<\infty\right\} ;
$$

(c) if $\sup _{n \in \mathbb{N}} \operatorname{Re}\left(\lambda_{n}\right)<\infty$, then, for every $t \geq 0, A(t)$ is the infinitesimal generator of a $C_{0}$-quasi-semigroup $R(t, s)$ given by

$$
R(t, s) x=\sum_{n=1}^{\infty} e^{\lambda_{n}(g(t+s)-g(t))}\left\langle x, \varphi_{n}\right\rangle \phi_{n}
$$

where $g(t)=\int_{0}^{t} a(\xi) d \xi ;$

(d) the growth bound of the quasi-semigroup at $t$ is given by

$$
\omega_{0}(t)=\inf _{s>0}\left(\frac{1}{s} \log \|R(t, s)\|\right)=a(t) \sup _{n \in \mathbb{N}} \operatorname{Re}\left(\lambda_{n}\right) .
$$

Proof. Proofs of (a) and (b) follow the proofs of Theorem 2.3.5 of [21] replacing $(\lambda I-A)^{-1}$ and $y_{N}$ with

$$
\begin{aligned}
(\lambda I-A(t))^{-1} & :=\sum_{n=1}^{\infty} \frac{1}{\lambda-a(t) \lambda_{n}}\left\langle x, \varphi_{n}\right\rangle \phi_{n}, \\
y_{N}(t) & :=\sum_{n=1}^{N} \frac{1}{\lambda-a(t) \lambda_{n}}\left\langle x, \varphi_{n}\right\rangle \phi_{n},
\end{aligned}
$$

respectively, for every $t \geq 0$. In this context $\mathscr{R}(\lambda, A(t))=(\lambda I-$ $A(t))^{-1}$. (c) Let $\omega=\sup _{n \geq 1} \operatorname{Re}\left(\lambda_{n}\right)$. Given $t \geq 0$ fixed, for $\lambda$ such that $\operatorname{Re}(\lambda)>a(t) \omega$, from (a)

$$
(\lambda I-A(t))^{-1} x=\sum_{n=1}^{\infty} \frac{1}{\lambda-a(t) \lambda_{n}}\left\langle x, \varphi_{n}\right\rangle \phi_{n}
$$

and by iteration we have

$$
(\lambda I-A(t))^{-r} x=\sum_{n=1}^{\infty} \frac{1}{\left(\lambda-a(t) \lambda_{n}\right)^{r}}\left\langle x, \varphi_{n}\right\rangle \phi_{n} .
$$

So by the condition $b$ of Lemma 2.3.2 of [21] for $m=h$ and $M=H$, we have

$$
\begin{aligned}
& \left\|(\lambda I-A(t))^{-r} x\right\|^{2} \\
& \leq \frac{H}{a(t)} \sum_{n=1}^{\infty} \frac{1}{\left|\lambda-a(t) \lambda_{n}\right|^{2 r}}\left|\left\langle x, \varphi_{n}\right\rangle\right|^{2} \\
& \quad \leq \frac{H}{h} \frac{\|x\|^{2}}{(\operatorname{Re}(\lambda)-a(t) \omega)^{2 r}} .
\end{aligned}
$$

Theorem 3.7 of [15] implies that $A(t)$ is an infinitesimal generator of a $C_{0}$-quasi-semigroup $R(t, s)$ with

$$
\|R(t, s)\| \leq \sqrt{\frac{H}{h}} e^{a(t) \omega s} .
$$

We verify that the operators $R(t, s), t, s \geq 0$, given by

$$
R(t, s) x=\sum_{n=1}^{\infty} e^{\lambda_{n}(g(t+s)-g(t))}\left\langle x, \varphi_{n}\right\rangle \phi_{n} \quad \forall x \in X,
$$

where $g(t)=\int_{0}^{t} a(\xi) d \xi$ and $\sup _{n \in \mathbb{N}} \operatorname{Re} \lambda_{n}<\infty$, are a $C_{0^{-}}$ quasi-semigroup on $X$ satisfying (15) with the infinitesimal generator

$$
A(t) x=a(t) \sum_{n=1}^{\infty} \lambda_{n}\left\langle x, \varphi_{n}\right\rangle \phi_{n}
$$

on domain

$$
\mathscr{D}=\left\{x \in X: \sum_{n=1}^{\infty}\left|\lambda_{n}\left\langle x, \varphi_{n}\right\rangle\right|^{2}<\infty\right\} .
$$

(d) By (10) we have

$$
\omega_{0}(t)=\inf _{s>0}\left(\frac{1}{s} \log \|R(t, s)\|\right) \leq a(t) \sup _{n \geq 1} \operatorname{Re}\left(\lambda_{n}\right) .
$$

On the other hand, taking $x=\phi_{n}$ in (16) we get

$$
\frac{1}{s} \log \|R(t, s)\|=\frac{1}{s}(g(t+s)-g(t))\left|\operatorname{Re}\left(\lambda_{n}\right)\right|
$$

$\forall n \in \mathbb{N}$

It implies

$$
\omega_{0}(t)=\inf _{s>0}\left(\frac{1}{s} \log \|R(t, s)\|\right) \geq a(t) \sup _{n \geq 1} \operatorname{Re}\left(\lambda_{n}\right) .
$$


Therefore

$$
\omega_{0}(t)=\inf _{s>0}\left(\frac{1}{s} \log \|R(t, s)\|\right)=a(t) \sup _{n \in \mathbb{N}} \operatorname{Re}\left(\lambda_{n}\right) .
$$

Corollary 4. If, for every $t \geq 0, A(t)$ is the generalized Rieszspectral generator of a $C_{0}$-quasi-semigroup $R(t, s)$ on a Hilbert space $X$, then for any $x_{0} \in \mathscr{D}$ and $r \geq 0$ the initial value problem

$$
\dot{x}(t)=A(r+t) x(t), \quad x(0)=x_{0}
$$

admits a unique solution.

Proof. It follows from Theorem 2.2 of [13] that (23) admits a unique solution.

\section{Nonautonomous Riesz-Spectral Systems}

In this section we shall apply the generalized Riesz-spectral operator in the linear nonautonomous control system $(A(t), B(t), C(t))$ of (1)-(2), where $A(t)$ is the generalized Riesz-spectral operator generating a $C_{0}$-quasi-semigroup $R(t, s)$ on $X$. In the sequel, we assume that the two requested real numbers $p$ and $q$ always satisfy

$$
\frac{1}{p}+\frac{1}{q}=1
$$

and $1<p<\infty$, unless specified.

Definition 5. Assume that the state linear system $(A(t)$, $B(t),-)$ holds for all initial state $x_{0} \in X$ and for all input $u \in L_{p}\left(\mathbb{R}^{+}, U\right)$. The state

$$
\begin{aligned}
x(t)=R(0, t) x_{0}+\int_{0}^{t} R(s, t-s) B(s) u(s) d s, & \\
& 0 \leq t<\infty,
\end{aligned}
$$

is defined to be a mild solution of (1).

We verify that $x \in \mathscr{C}\left(\mathbb{R}^{+}, X\right)$ and the output $y$ defined by (2) always belongs to $L_{q}\left(\mathbb{R}^{+}, Y\right)$. The definitions of the controllability and observability in this paper follow the definitions for the autonomous case; see, for example, [21].

Definition 6. The linear system $(A(t), B(t),-)$ is said to be

(a) exactly controllable on $[0, \tau]$ if for each $x_{0}, x_{1} \in X$ there exists a control $u \in L_{p}([0, \tau], U)$ such that the mild solution $x(\cdot)$ of (1) corresponding to $u(\cdot)$ satisfies $x(\tau)=x_{1}$

(b) approximately controllable on $[0, \tau]$ if for each $x_{0}, x_{1} \in X$ and any $\epsilon>0$ there exists a control $u \in L_{p}([0, \tau], U)$ such that the mild solution $x(\cdot)$ of (1) corresponding to $u(\cdot)$ satisfies $\left\|x(\tau)-x_{1}\right\|<\epsilon$.
A controllability map of $(A(t), B(t),-)$ on $[0, \tau]$ is a bounded linear map $\mathscr{B}_{\tau}: L_{p}([0, \tau] ; U) \rightarrow X$ defined by

$$
\mathscr{B}_{\tau} u=\int_{0}^{\tau} R(s, \tau-s) B(s) u(s) d s .
$$

It is easy to show that the system $(A(t), B(t),-)$ is exactly controllable on $[0, \tau]$ if and only if ran $\mathscr{B}_{\tau}=X$, where ran $T$ denotes the range of $T$. Also, system $(A(t), B(t),-)$ is approximately controllable on $[0, \tau]$ if and only if $\overline{\operatorname{ran} \mathscr{B}_{\tau}}=$ $X$.

Lemma 7. The controllability map in (26) satisfies the following conditions.

(a) The operator $\mathscr{B}_{\tau} \in \mathscr{L}\left(L_{p}([0, \tau], U), X\right)$ and $\mathscr{B}_{t} \in$ $\mathscr{L}\left(L_{p}([0, \tau], U), L_{p}([0, \tau], X)\right)$ for $0 \leq t \leq \tau$.

(b) $\left(\mathscr{B}_{\tau}^{*} x\right)(s)=B^{*}(s) R^{*}(s, \tau-s) x$ on $[0, \tau]$.

Proof. (a) Since $R(t, s)$ is strongly continuous and $u \in$ $L_{p}([0, \tau], U)$, then the map $s \mapsto\langle x, R(s, \tau-s) B(s) u(s)\rangle$ is measurable on $[0, \tau]$ for every $x \in X$. Moreover,

$$
\begin{aligned}
\int_{0}^{\tau} & \|R(s, \tau-s) B(s) u(s)\|_{X} d s \\
& \leq \int_{0}^{\tau} M(\tau-s)\|B(\cdot)\|_{\mathscr{L}(U, X)}\|u\|_{L_{p}} d s \\
& \leq \tau M(\tau)\|B(\cdot)\|_{\mathscr{L}(U, X)}\|u\|_{L_{p}}<\infty .
\end{aligned}
$$

Lemma A.5.5 of [21] states that the integral in (26) is welldefined. We verify easily that $\mathscr{B}_{\tau}$ is linear. Now, for $0 \leq t \leq \tau$ we have

$$
\begin{aligned}
\left\|\mathscr{B}_{t} u\right\| & \leq \int_{0}^{t}\|R(s, \tau-s) B(s) u(s)\|_{X} d s \\
& \leq \int_{0}^{t} M(t-s)\|B(\cdot)\|_{\mathscr{L}(U, X)}\|u\|_{L_{p}} d s \\
& \leq t M(t)\|B(\cdot)\|_{\mathscr{L}(U, X)}\|u\|_{L_{p}} .
\end{aligned}
$$

This shows that $\mathscr{B}_{\tau}$ is a bounded mapping from $L_{p}([0, \tau], U)$ to $X$ and $u \mapsto \mathscr{B}_{t} u, 0 \leq t \leq \tau$, is a bounded mapping from $L_{p}([0, \tau], U)$ to $L_{p}([0, \tau], X)$.

(b) The definition of adjoint operator shows that $\mathscr{B}_{\tau}^{*}$ is bounded. Moreover,

$$
\begin{aligned}
\left\langle u, \mathscr{B}_{\tau}^{*} x\right\rangle_{L_{p}} & =\left\langle\mathscr{B}_{\tau} u, x\right\rangle_{X} \\
& =\left\langle\int_{0}^{\tau} R(s, \tau-s) B(s) u(s) d s, x\right\rangle_{X} \\
& =\int_{0}^{\tau}\langle R(s, \tau-s) B(s) u(s), x\rangle_{X} d s \\
& =\int_{0}^{\tau}\left\langle u(s), B^{*}(s) R^{*}(s, \tau-s) x\right\rangle_{U} d s \\
& =\left\langle u, B^{*}(\cdot) R^{*}(\cdot, \tau-\cdot) x\right\rangle_{L_{p}} .
\end{aligned}
$$

This proves that $\mathscr{B}_{\tau}^{*} x=B^{*}(s) R^{*}(s, \tau-s) x$ on $[0, \tau]$. 
Theorem 8. For $u \in L_{p}([0, \tau], U)$, the system $(A(t), B(t),-)$ is exactly controllable on $[0, \tau]$ if and only if any one of the following conditions holds for some $\gamma>0$ and all $x \in X$ :

(a) $\gamma\left\|B^{*}(\cdot) R^{*}(\cdot, \tau-\cdot) x\right\|_{L_{p}} \geq\|x\|$.

(b) $\operatorname{ker} \mathscr{B}_{\tau}^{*}=\{0\}$ and ran $\mathscr{B}_{\tau}^{*}$ is closed.

Proof. (a) We set $V=L_{p}([0, \tau], U)$, so $\mathscr{B}_{\tau} \in \mathscr{L}(V, X)$. It is enough to prove that ran $\mathscr{B}_{\tau}=X$. By similarity of adjoint and dual operator in Hilbert space, Corollary 3.5 of [20] states

$$
\operatorname{ran} \mathscr{B}_{\tau}=X
$$

if and only if there exists $\gamma>0$ such that

$$
\gamma\left\|\mathscr{B}_{\tau}^{*} x\right\|_{L_{p}} \geq\|x\|
$$

for all $x \in X$. So, by condition (b) of Lemma 7 the assertion is confirmed.

(b) The condition $\gamma\left\|\mathscr{B}_{\tau}^{*} x\right\|_{L_{p}} \geq\|x\|$ shows that $\mathscr{B}_{\tau}^{*}$ is injective, and so $\operatorname{ker} \mathscr{B}_{\tau}^{*}=\{0\}$. Next, let $\left(\mathscr{B}_{\tau}^{*} x_{n}\right)$ be a Cauchy sequence in $L_{p}([0, \tau], U)$. Condition (a) shows that $\left(x_{n}\right)$ is a Cauchy sequence in $X$. However, Lemma 7 (a) forces $\mathscr{B}_{\tau}^{*} x_{n} \rightarrow \mathscr{B}_{\tau}^{*} x$ for some $x \in \mathscr{B}_{\tau}^{*}$. Thus, $\mathscr{B}_{\tau}^{*}$ has a closed range.

Theorem 9. The linear system $(A(t), B(t),-)$ is approximately controllable on $[0, \tau]$ if and only if any one of the following conditions holds:

(a) $B^{*}(s) R^{*}(s, \tau-s) x=0,0 \leq s \leq \tau$, implies $x=0$.

(b) $\operatorname{ker} \mathscr{B}_{\tau}^{*}=\{0\}$.

Proof. (a) We see that the system $(A(t), B(t),-)$ is approximately controllable on $[0, \tau]$ if and only if $\overline{\operatorname{ran} \mathscr{B}_{\tau}}=X$. According to Lemma VI 2.8 of [24], this is equivalent to the fact that the mapping $\mathscr{B}_{\tau}^{\prime}: X^{\prime} \rightarrow L_{q}\left([0, \tau], U^{\prime}\right)$ is injective. The similarity between adjoint and dual operator gives

$$
B^{*}(\cdot) R^{*}(\cdot, \tau-\cdot) x=0
$$

which implies $x=0$ almost everywhere. Therefore, if

$$
B^{*}(s) R^{*}(s, \tau-s) x=0, \quad 0 \leq s \leq \tau,
$$

this verifies that $x=0$.

(b) Condition (a) and condition (b) of Lemma 7 give the desired result.

Complementary to Definition 6, we define the exact observability and the approximate observability as follows.

Definition 10. The linear system $(A(t), B(t), C(t))$ is said to be

(a) exactly observable on $[0, \tau]$ if the initial state can be uniquely constructed from the knowledge of the output in $L_{q}([0, \tau], Y)$;

(b) approximately observable on $[0, \tau]$ if the knowledge of the output in $L_{q}([0, \tau], Y)$ determines the initial state uniquely.
The observability map of the system $(A(t), B(t), C(t))$ on $[0, \tau]$ is a bounded linear map $\mathscr{C}_{\tau}: X \rightarrow L_{q}([0, \tau] ; Y)$ defined by

$$
\mathscr{C}_{\tau} x=C(t) R(0, t) x
$$

for $0 \leq t \leq \tau$.

From Definition 10 and the definition of observability map we verify that the system $(A(t), B(t), C(t))$ is exactly observable on $[0, \tau]$ if and only if $\mathscr{C}_{\tau}$ is injective and its inverse is bounded on ran $\mathscr{C}_{\tau}$. Also, $(A(t), B(t), C(t))$ is approximately observable on $[0, \tau]$ if and only if ker $\mathscr{C}_{\tau}=\{0\}$.

Lemma 11. For the linear system $(A(t), B(t), C(t))$ one has the following duality:

(a) The linear system $(A(t),-, C(t))$ is approximately observable on $[0, \tau]$ if and only if the dual $\left(A^{*}(t)\right.$, $\left.C^{*}(t),-\right)$ is approximately controllable on $[0, \tau]$.

(b) The linear system $(A(t),-, C(t))$ is exactly observable on $[0, \tau]$ if and only if the dual $\left(A^{*}(t), C^{*}(t),-\right)$ is exactly controllable on $[0, \tau]$.

Proof. As a consequence of Proposition 1.2 and Theorem 1.6 of [14], if $A(t)$ generates a $C_{0}$-quasi-semigroup $R(t, s)$ on a Hilbert space, then $A^{*}(t)$ generates the $C_{0}$-quasi-semigroup $R^{*}(t, s)$. Furthermore, we verify that

$$
\mathscr{C}_{\tau}^{*} y=\int_{0}^{\tau} R^{*}(0, s) C^{*}(s) y(s) d s .
$$

This implies that the range of $\mathscr{C}_{\tau}^{*}$ equals that of the controllability map for the dual system $\left(A^{*}(t), C^{*}(t),-\right)$. If $\mathscr{B}_{\tau}$ denotes the controllability map of the dual system, then $\mathscr{C}_{\tau}^{*}=\mathscr{B}_{\tau}$ or $\mathscr{C}_{\tau}=\mathscr{B}_{\tau}^{*}$.

(a) We see that $(A(t),-, C(t))$ is approximately observable on $[0, \tau]$ if and only if $\{0\}=\operatorname{ker} \mathscr{C}_{\tau}=\operatorname{ker} \mathscr{B}_{\tau}^{*}$. Condition (b) of Theorem 9 implies that ker $\mathscr{B}_{\tau}^{*}=\{0\}$ if and only if $\left(A^{*}(t), C^{*}(t),-\right)$ is approximately controllable on $[0, \tau]$. This proves the equivalence.

(b) Suppose that $(A(t),-, C(t))$ is exactly observable on $[0, \tau]$. There exists an inverse $\mathscr{C}_{\tau}^{-1}$ on ran $\mathscr{C}_{\tau}$ and a constant $\kappa>0$ such that

$$
\|x\|_{X}=\left\|\mathscr{C}_{\tau}^{-1} \mathscr{C}_{\tau} x\right\| \leq \kappa\left\|\mathscr{C}_{\tau} x\right\|=\kappa\left\|\mathscr{B}_{\tau}^{*}\right\|
$$

The exact controllability of $\left(A^{*}(t), C^{*}(t),-\right)$ follows from Theorem 8.

Conversely, assume that $\left(A^{*}(t), C^{*}(t),-\right)$ is exactly controllable on $[0, \tau]$. Theorem 8 (a) gives that $\mathscr{B}_{\tau}^{*}$ is injective and ran $\mathscr{B}_{\tau}^{*}$ is closed. Since $\mathscr{B}_{\tau}^{*}=\mathscr{C}_{\tau}$, then $\mathscr{C}_{\tau}$ is injective and $\operatorname{ran} \mathscr{C}_{\tau}$ is closed. This states that $(A(t),-, C(t))$ is exactly observable on $[0, \tau]$.

Theorems 8 and 9 and Lemma 11 yield the following conditions for observability.

Corollary 12. For the linear system $(A(t),-, C(t))$, one has the following necessary and sufficient conditions for exact and approximate observability: 
(a) $(A(t),-, C(t))$ is exactly observable on $[0, \tau]$ if and only if any one of the following conditions holds for some $\gamma>$ 0 and all $x \in X$ :

(i) $\gamma\|C(\cdot) R(0, \cdot) x\|_{L_{q}} \geq\|x\|$.

(ii) $\operatorname{ker} \mathscr{C}_{\tau}=\{0\}$ and $\operatorname{ran} \mathscr{C}_{\tau}$ is closed.

(b) $(A(t),-, C(t))$ is approximately observable on $[0, \tau]$ if and only if any one of the following conditions holds:

(i) $C(s) R(0, s) x=0,0 \leq s \leq \tau$, implies $x=0$.

(ii) $\operatorname{ker} \mathscr{C}_{\tau}=\{0\}$.

In the infinite-dimensional system, it is generally easier to prove the approximate controllability and approximate observability than the exact controllability and exact observability. Next, we shall derive easily verifiable criteria for the approximate controllability and approximate observability of the generalized Riesz-spectral systems with finite-rank inputs and outputs.

Consider system (1)-(2) with finite-rank inputs and outputs

$$
\begin{aligned}
\dot{x}(t) & =A(t) x(t)+\sum_{i=1}^{m} b_{i}(t) u_{i}(t), \\
t \geq 0, x(0)=x_{0}, & t \geq \\
y(t) & =\left(\left\langle x(t), c_{1}(t)\right\rangle, \ldots,\left\langle x(t), c_{k}(t)\right\rangle\right)^{\operatorname{tr}},
\end{aligned}
$$

where $A(t)$ is the generalized Riesz-spectral operator of (4), $b_{i}(t) \in X, i=1, \ldots, m, c_{i}(t) \in X, i=1, \ldots, k$, and $u_{i} \in$ $L_{p}\left(\mathbb{R}^{+}\right), 1<p<\infty$. The symbol $S^{\text {tr }}$ denotes the transpose of $S$. If we set $u=\left(u_{1}, \ldots, u_{m}\right) \in U:=\mathbb{R}^{m}, B(t) u:=\sum_{i=1}^{m} b_{i}(t) u_{i}$, then $u \in L_{p}\left(\mathbb{R}^{+}, U\right)$. In this case we have

$$
C(t) x(t)=\left(\left\langle x(t), c_{1}(t)\right\rangle, \ldots,\left\langle x(t), c_{k}(t)\right\rangle\right)^{\mathrm{tr}} .
$$

Let $A$ be the Riesz-spectral operator with simple eigenvalues $\left\{\lambda_{n}: n \in \mathbb{N}\right\}$ and corresponding eigenvectors $\left\{\phi_{n}: n \in \mathbb{N}\right\}$. In addition, if $\left\{\varphi_{n}: n \in \mathbb{N}\right\}$ are the eigenvectors of $A^{*}$ such that $\left\langle\phi_{n}, \varphi_{m}\right\rangle=\delta_{m n}$ and $\sup _{n \in \mathbb{N}} \operatorname{Re}\left(\lambda_{n}\right)<\infty$, then according to the condition (c) of Theorem $3 A(t)$ is the infinitesimal generator of a $C_{0}$-quasi-semigroup $R(t, s)$ given by

$$
R(t, s) x=\sum_{n=1}^{\infty} e^{\lambda_{n}(g(t+s)-g(t))}\left\langle x, \varphi_{n}\right\rangle \phi_{n},
$$

where $g(t)=\int_{0}^{t} a(\xi) d \xi$ and $A(t)$ is the form of (4). In this context we verify that

$$
\begin{aligned}
B^{*}(t) x & =\left(\left\langle b_{1}(t), x\right\rangle_{X}, \ldots,\left\langle b_{m}(t), x\right\rangle_{X}\right), \\
R^{*}(t, s) x & =\sum_{n=1}^{\infty} e^{\overline{\lambda_{n}}(g(t+s)-g(t))}\left\langle x, \phi_{n}\right\rangle \varphi_{n} .
\end{aligned}
$$

By Theorem 9, system (37) is approximately controllable on $[0, \tau]$ if and only if

$$
\begin{aligned}
\sum_{n=1}^{\infty} e^{\overline{\lambda_{n}}(g(\tau)-g(t))}\left\langle x, \phi_{n}\right\rangle\left\langle b_{i}(t), \varphi_{n}\right\rangle & =0, \\
& i=1, \ldots, m, 0 \leq t \leq \tau
\end{aligned}
$$

implies that $x=0$.
Next, we have

$$
C(t) R(0, t) x=\sum_{n=1}^{\infty} e^{\lambda_{n}(g(t))}\left\langle x, \varphi_{n}\right\rangle C(t) \phi_{n} .
$$

In virtue of Corollary 12, system (37)-(38) is approximately observable on $[0, \tau]$ if and only if

$$
\sum_{n=1}^{\infty} e^{\lambda_{n}(g(t))}\left\langle x, \varphi_{n}\right\rangle C(t) \phi_{n}=0
$$

implies that $x=0$.

These two facts deal with the following theorem which is a generalization of Theorem 4.2 .3 of [21] for the autonomous case.

Theorem 13. Consider the linear system $(A(t), B(t), C(t))$ of (37)-(38), where $A$ is a Riesz-spectral operator with simple eigenvalues $\left\{\lambda_{n}: n \in \mathbb{N}\right\}$ such that $\sup _{n \in \mathbb{N}} \operatorname{Re}\left(\lambda_{n}\right)<\infty$ and corresponding eigenvectors $\left\{\phi_{n}: n \in \mathbb{N}\right\}$. Let $\left\{\varphi_{n}: n \in \mathbb{N}\right\}$ be the eigenvectors of $A^{*}$ such that $\left\langle\phi_{n}, \varphi_{m}\right\rangle=\delta_{m n}$. Then

(a) $(A(t), B(t),-)$ is approximately controllable on $[0, \tau]$ if and only if for all $n$

$$
\operatorname{rank}\left(\left\langle b_{1}(t), \varphi_{n}\right\rangle, \ldots,\left\langle b_{m}(t), \varphi_{n}\right\rangle\right)=1
$$

for all $t \in[0, \tau]$;

(b) $(A(t),-, C(t))$ is approximately observable on $[0, \tau]$ if and only if for all $n$

$$
\operatorname{rank}\left(\left\langle\phi_{n}, c_{1}(t)\right\rangle, \ldots,\left\langle\phi_{n}, c_{k}(t)\right\rangle\right)=1
$$

for all $t \in[0, \tau]$.

Proof. (a) We consider the matrix $B_{n}$ :

$$
B_{n}=\left(\left\langle b_{1}(t), \varphi_{n}\right\rangle, \ldots,\left\langle b_{m}(t), \varphi_{n}\right\rangle\right)
$$

on $[0, \tau]$. By Lemma 3.14 of [20] and $(42),(A(t), B(t),-)$ is approximately controllable on $[0, \tau]$ if and only if for all $n$

$$
\left\langle x, \phi_{n}\right\rangle\left\langle b_{i}(t), \varphi_{n}\right\rangle=0, \quad i=1, \ldots, m, 0 \leq t \leq \tau
$$

implies $x=0$. Suppose that $(A(t), B(t),-)$ is not approximately controllable on $[0, \tau]$, there exists an $n \in \mathbb{N}$ such that $\left\langle x, \phi_{n}\right\rangle \neq 0$ and

$$
\left\langle x, \phi_{n}\right\rangle\left\langle b_{i}(t), \varphi_{n}\right\rangle=0, \quad i=1, \ldots, m, 0 \leq t \leq \tau .
$$

This gives $\left\langle b_{i}(t), \varphi_{n}\right\rangle=0$ for all $i=1, \ldots, m$ and $t \in[0, \tau]$, and so rank $B_{n} \neq 1$.

Conversely, suppose that $\operatorname{rank} B_{n_{0}} \neq 1$ for some $n_{0}$, then $\left\langle b_{i}(t), \phi_{n_{0}}\right\rangle=0$, for all $i=1, \ldots, m$ and $t \in[0, \tau]$. So we can find a nonzero $x \in X$ such that

$$
\left\langle x, \phi_{n_{0}}\right\rangle\left\langle b_{i}(t), \varphi_{n_{0}}\right\rangle=0 .
$$

Thus, (42) is satisfied for $x \neq 0$. This is equivalent to the fact that $(A(t), B(t),-)$ is not approximately controllable on $[0, \tau]$.

(b) We can have similar proof to (a) for the matrix

$$
C_{n}=\left(\left\langle\phi_{n}, c_{1}(t)\right\rangle, \ldots,\left\langle\phi_{n}, c_{k}(t)\right\rangle\right)
$$

on $[0, \tau]$. 


\section{Nonautonomous Sturm-Liouville Systems}

In this section we shall discuss nonautonomous SturmLiouville systems, the specifically nonautonomous Rieszspectral systems. First let us recall the definition of SturmLiouville operators. In the sequel, we set $X$ to be the Hilbert space of $L_{2}[a, b]$. Consider an operator $\mathscr{A}$ on its domain

$$
\begin{aligned}
& \mathscr{D}(\mathscr{A})=\{x \\
& \in X: x, \frac{d x}{d \xi} \text { are absolutely continuous, } \frac{d^{2} x}{d \xi^{2}} \\
& \quad \in X, a_{1} \frac{d x}{d \xi}(a)+a_{2} x(a)=0, b_{1} \frac{d x}{d \xi}(b)+b_{2} x(b) \\
& =0\},
\end{aligned}
$$

where $\left(a_{1}, a_{2}\right) \neq(0,0)$ and $\left(b_{1}, b_{2}\right) \neq(0,0)$. Operator $\mathscr{A}$ is called a Sturm-Liouville operator if

$$
\mathscr{A} x:=\frac{1}{w(\xi)}\left(-\frac{d}{d \xi}\left(p(\xi) \frac{d x}{d \xi}(\xi)\right)+q(\xi) x(\xi)\right),
$$

for $x \in \mathscr{D}(\mathscr{A})$, where $w, p, q$, and $d p / d \xi$ are real-valued continuous functions on $[a, b]$ such that $p(\xi)>0$ and $w(\xi)>$ 0 .

Since $a$ and $b$ are finite, the definition only corresponds to regular Sturm-Liouville problems. We verify that $\mathscr{A}$ is a selfadjoint operator with real, countable, and simple eigenvalues $\lambda_{n}$ such that $0<\lambda_{1}<\lambda_{2}<\cdots($ see $[25,26])$.

We define a nonautonomous Sturm-Liouville operator to be an operator of form (4):

$$
A(t)=a(t) \mathscr{A}, \quad t \geq 0,
$$

where $\mathscr{A}$ is a Sturm-Liouville operator on its domain $\mathscr{D}(\mathscr{A})$ given by (52).

Definition 14. The state linear system $(A(t), B(t), C(t))$ of $(1)$ (2) is called a nonautonomous Sturm-Liouville system if $A(t)$ is the negative of a nonautonomous Sturm-Liouville operator of form (54).

Corollary 15. For every $t \geq 0$, let $A(t)$ be the negative of a nonautonomous Sturm-Liouville operator of the form (54) on its domain $\mathscr{D}(A)$ given by (52). Then
(a) $A(t)$ is generalized Riesz-spectral operator;
(b) $A(t)$ is the infinitesimal generator of a $C_{0}$-quasi- semigroup on $X$.

Proof. (a) Lemma 1 of [27] gives the fact that $A(t)$ is generalized Riesz-spectral operator.

(b) If $\left\{\lambda_{n}: n \in \mathbb{N}\right\}$ is the set of eigenvalues of $-\mathscr{A}$, then $\sup _{n \in \mathbb{N}} \operatorname{Re}\left(\lambda_{n}\right)<\infty$. Hence, Theorem 3 concludes that, for every $t \geq 0, A(t)$ is the infinitesimal generator of a $C_{0}$-quasisemigroup on $X$.
We note that Corollary 15 does not hold when $A(t)$ is a nonautonomous Sturm-Liouville operator. Indeed, $A(t)=$ $-a(t)\left(d^{2} / d \xi^{2}\right)$ is a nonautonomous Sturm-Liouville operator, but it does not generate any $C_{0}$-quasi-semigroup (see Section 3 [14]). Corollary 15 also concludes that any nonautonomous Sturm-Liouville system is the nonautonomous Riesz-spectral system. Therefore, all of the results of the controllability and observability in the previous section are applicable on the nonautonomous Sturm-Liouville systems.

\section{Applications}

In this section, we consider two examples of applications to confirm the results of the generalized Riesz-spectral operator in the nonautonomous systems.

Example 1. Consider the boundary condition problem of the PDE:

$$
\begin{aligned}
& \frac{\partial x}{\partial t}(t, \xi)=\frac{\xi^{2}}{t+1} \frac{\partial^{2} x}{\partial \xi^{2}}(t, \xi)+\frac{\xi}{t+1} \frac{\partial x}{\partial \xi}(t, \xi), \\
& \quad 1<\xi<b, t \geq 0 \\
& x(t, 1)=x(t, b)=0, \quad 1<b<\infty .
\end{aligned}
$$

We are ready to show that the problem has a unique solution. Let $X$ be a Hilbert space of $L_{2}[1, b]$. Problem (55) can be written as

$$
\dot{x}(t)=A(t) x(t), \quad t \geq 0,
$$

on $X$, where $A(t):=a(t) A, a(t)=1 /(t+1)$, and

$$
A x(\xi):=\xi^{2} \frac{d^{2} x}{d \xi^{2}}+\xi \frac{d x}{d \xi}
$$

on $\mathscr{D}$ with

$$
\begin{aligned}
\mathscr{D} & =\{x \\
& \in X: x, \frac{d x}{d \xi} \text { are absolutely continuous, } \frac{d^{2} x}{d \xi^{2}} \\
& \in X, x(1)=x(b)=0\} .
\end{aligned}
$$

We verify that operator $A$ is not self-adjoint on $\mathscr{D}$. Furthermore, we obtain the eigenvalues and corresponding eigenvectors of $A$ as

$$
\begin{aligned}
\lambda_{n} & =-\left(\frac{n^{2} \pi^{2}}{\log b}\right), \\
\phi_{n}(\xi) & =\sqrt{2} \sin (n \pi \log \xi)
\end{aligned}
$$

$$
\text { for } 1 \leq \xi \leq b \text {, }
$$

respectively. It is obvious that every eigenvalue $\lambda_{n}$ is simple and the set $\left\{\phi_{n}: n \in \mathbb{N}\right\}$ forms Riesz basis of $X$. Moreover, $\overline{\left\{\lambda_{n}, n \in \mathbb{N}\right\}}$ is totally disconnected, that is, for $c, d \in$ $\overline{\left\{\lambda_{n}, n \in \mathbb{N}\right\}},[c, d] \nsubseteq \overline{\left\{\lambda_{n}, n \in \mathbb{N}\right\}}$. 
The adjoint of $A$ is

$$
A^{*} x(\xi):=\xi^{2} \frac{d^{2} x}{d \xi^{2}}+3 \xi \frac{d x}{d \xi}+x(\xi)
$$

on $\mathscr{D}\left(A^{*}\right)=\mathscr{D}(A)$. The eigenvalues and corresponding eigenvectors of $A^{*}$ are

$$
\begin{aligned}
\mu_{n} & =-\left(\frac{n^{2} \pi^{2}}{\log b}\right), \\
\psi_{n}(\xi) & =\sqrt{2} \xi^{-1} \sin (n \pi \log \xi)
\end{aligned}
$$

for all $1 \leq \xi \leq b, n \in \mathbb{N}$, and satisfy

$$
\left\langle\phi_{m}, \psi_{n}\right\rangle=\delta_{m n} \quad \forall m, n \in \mathbb{N}
$$

Next, since the adjoint of any operator is always closed, then $A^{*}$ is closed. But in this case we have $A=\left(A^{*}\right)^{*}$, so $A$ is closed. Thus, $A$ is a Riesz-spectral operator. In other words, $A(t), t \geq 0$, is a generalized Riesz-spectral operator.

Since $\sup _{n \in \mathbb{N}} \operatorname{Re}\left(\lambda_{n}\right)=-\left(\pi^{2} / \log b\right)<\infty$, condition (c) of Theorem 3 forces that $A(t)$ is the infinitesimal generator of a $C_{0}$-quasi-semigroup $R(t, s)$ given by

$$
R(t, s) x=\sum_{n=1}^{\infty}\left(\frac{t+s+1}{t+1}\right)^{\lambda_{n}}\left\langle x, \psi_{n}\right\rangle \phi_{n} .
$$

Corollary 4 guarantees that for each $x_{0} \in \mathscr{D}$ problem (56) admits a unique solution

$$
x(t)=R(0, t) x_{0}, \quad x(0)=x_{0} .
$$

Thus, boundary condition problem (55) has a solution

$$
x(t, \xi)=\sum_{n=1}^{\infty}(t+1)^{\lambda_{n}}\left\langle x_{0}, \psi_{n}\right\rangle \phi_{n} .
$$

Example 2. Consider the controlled wave equation

$$
\begin{aligned}
& \frac{\partial^{2} x}{\partial t^{2}}(t, \xi)=\frac{\partial^{2} x}{\partial \xi^{2}}(t, \xi)+b(t) u(t, \xi) \\
& 0<\xi<1, t \geq 0 \\
& x(t, 0)=x(t, 1)=0,
\end{aligned}
$$

where $b: \mathbb{R}^{+} \rightarrow \mathbb{R}$ is bounded uniformly continuous and $u(t, \cdot) \in L_{2}[0,1]$ is a distributed control.

We shall analyze the approximate controllability and approximate observability of the system. Problem (66) can be formulated as a linear system:

$$
\dot{w}(t)=A(t) w(t)+B(t) u(t), \quad t \geq 0,
$$

on the Hilbert space $X=\mathscr{D}\left(A_{0}^{1 / 2}\right) \oplus L_{2}[0,1]$ with the inner product

$$
\langle v, y\rangle_{X}=\left\langle A_{0}^{1 / 2} v_{1}, A_{0}^{1 / 2} y_{1}\right\rangle_{L_{2}[0,1]}+\left\langle v_{2}, y_{2}\right\rangle_{L_{2}[0,1]},
$$

where $v=\left(\begin{array}{l}v_{1} \\ v_{2}\end{array}\right)$ and $y=\left(\begin{array}{l}y_{1} \\ y_{2}\end{array}\right)$. In this context $A(t)=\left(\begin{array}{cc}0 & I \\ -A_{0} & 0\end{array}\right)$, $B(t)=\left(\begin{array}{c}0 \\ b(t)\end{array}\right), w=\left(\begin{array}{c}x \\ d x / d t\end{array}\right)$, and $A_{0} h=-d^{2} h / d \xi^{2}$ for $h \epsilon$ $\mathscr{D}\left(A_{0}\right)$ with

$$
\begin{aligned}
& \mathscr{D}\left(A_{0}\right)=\{h \\
& \quad \in L_{2}[0,1]: h, \frac{d h}{d \xi} \text { are absolutely continuous } \frac{d^{2} h}{d \xi^{2}} \\
& \left.\quad \in L_{2}[0,1], h(0)=h(1)=0\right\} .
\end{aligned}
$$

We verify that $A(t)$ has the eigenvalues $\lambda_{n}=i n \pi, n=$ $\pm 1, \pm 2, \ldots$ and the corresponding Riesz basis of eigenvectors $\phi_{n}(\xi)=(1 / i n \pi)\left(\begin{array}{c}\sin (n \pi \xi) \\ i n \pi \sin (n \pi \xi)\end{array}\right)$, where $i=\sqrt{-1}$. We see that $\phi_{n}(\xi)=\psi_{n}(\xi)$ for every $n$. Moreover, Example 2.3.8 of [21] shows that $A(t)$ is a Riesz-spectral operator on $X$. Hence, $A(t)$ is the generalized Riesz-spectral operator generating a $C_{0^{-}}$ quasi-semigroup $R(t, s)$.

We assume that the system is controlled around the point $\xi_{c}$. So, we may set

$$
u(t, \xi)=u(t) \frac{1}{2 \epsilon} \chi_{\left[\xi_{c}-\epsilon, \xi_{c}+\epsilon\right]}(\xi)
$$

where $\chi$ is an indicator function. Theorem 13 shows that system (67) is approximately controllable on $[0, \tau]$ if and only if

$$
\int_{0}^{1} b(t) \sin (n \pi \xi) d \xi=\frac{b(t)}{n \pi \epsilon} \sin \left(n \pi \xi_{c}\right) \sin (n \pi \epsilon) \neq 0
$$

for $n \geq 1$.

Equation (71) demonstrates that the control points $\xi_{c}$ for which $\sin \left(n \pi \xi_{c}\right)=0$ affect the loss of approximate controllability. This is also the case when $b(t)=0$, that is, at the zeros of $b$ on interval $[0, \tau]$.

Next, consider the observation

$$
y(t)=\int_{0}^{1} c(t, \xi) x(t, \xi) d \xi,
$$

where $c(t, \xi)=(1 / 2 v) c(t) \chi_{\left[\xi_{s}-v, \xi_{s}+v\right]}(\xi)$ is an output function around the sensing point $\xi_{s}$. Following Example 4.2.5 of [21] we can reformulate the observation map as an inner product on $X$ :

$$
\begin{aligned}
C(t)\left(\begin{array}{c}
v_{1} \\
v_{2}
\end{array}\right):=\int_{0}^{1} c(t, \xi) v_{1}(\xi) d \xi=\left\langle v_{1}, c(t, \xi)\right\rangle_{L_{2}[0,1]} \\
=\left\langle\left(\begin{array}{c}
v_{1} \\
v_{2}
\end{array}\right),\left(\begin{array}{c}
c(t) k \\
0
\end{array}\right)\right\rangle_{X}, \\
\text { where } k=A_{0}^{-1} \frac{1}{2 v} \chi_{\left[\xi_{s}-v, \xi_{s}+v\right]} .
\end{aligned}
$$

Condition (b) of Theorem 13 gives the fact that $(A(t),-, C(t))$ is approximately observable on $[0, \tau]$ if and only if for all $n$

$$
\left\langle\phi_{n},\left(\begin{array}{c}
c(t) k \\
0
\end{array}\right)\right\rangle_{X} \neq 0 .
$$


We verify that

$$
\begin{aligned}
& \left\langle\phi_{n},\left(\begin{array}{c}
c(t) k \\
0
\end{array}\right)\right\rangle_{X} \\
& \quad=\frac{1}{i n \pi}\left\langle\sin (n \pi \cdot), \frac{c(t)}{2 \nu} \chi_{\left[\xi_{s}-v, \xi_{s}+v\right]}(\cdot)\right\rangle_{L_{2}[0,1]} .
\end{aligned}
$$

Therefore, the system is approximately observable on $[0, \tau]$ if and only if for all $n$

$$
\begin{aligned}
& c(t) \int_{\xi_{s}-v}^{\xi_{s}+v} \sin (n \pi \xi) d \xi=\frac{c(t)}{n \pi \nu} \sin \left(n \pi \xi_{s}\right) \sin (n \pi \nu) \\
& \quad \neq 0 .
\end{aligned}
$$

This shows that the system loses the approximate observability at points for which $\sin \left(n \pi \xi_{s}\right)=0$ for some $n$ or $c(t)=0$. Specially, for $c(t)=\sin \left(f_{s}(t) \pi\right)$, where $f_{s}(t)$ is the sampling frequency, then the system loses the approximate observability at sampling frequencies of $f_{s}=k$, for $k=$ $0,1,2, \ldots$ discrete measurement.

Example 3. Consider the controlled nonautonomous heat equation on interval $[1, b]$ :

$$
\begin{aligned}
\frac{\partial x}{\partial t}(t, \xi) & =a(t) \frac{\partial}{\partial \xi}\left(\xi^{2} \frac{\partial x}{\partial \xi}(t, \xi)\right)+\beta(t) u(t, \xi) \\
x(t, 1) & =x(t, b)=0,
\end{aligned}
$$

where $a, \beta: \mathbb{R}^{+} \rightarrow \mathbb{R}$ are bounded uniformly continuous and $u(t, \cdot) \in L_{2}[1, b]$ is a distributed control.

We shall analyze the approximate controllability and approximate observability of the system. Let $X$ be a Hilbert space of $L_{2}[1, b]$. System (77) can be formulated as a nonautonomous Sturm-Liouville system

$$
\dot{x}(t)=A(t) x(t)+B(t) u(t), \quad t \geq 0
$$

on $X$, where $A(t):=-a(t) \mathscr{A}, B(t):=\beta(t) I$, and $\mathscr{A}$ is a SturmLiouville operator (53) with $w(\xi)=1, p(\xi)=\xi^{2}$, and $q(\xi)=0$ on its domain $\mathscr{D}$ :

$$
\begin{aligned}
\mathscr{D} & =\{x \\
& \in X: x, \frac{d x}{d \xi} \text { are absolutely continuous, } \frac{d^{2} x}{d \xi^{2}} \\
& \in X, x(1)=x(b)=0\} .
\end{aligned}
$$

The eigenvalues and corresponding eigenvectors of $-\mathscr{A}$ are

$$
\begin{aligned}
\lambda_{n} & =-\left(\frac{n^{2} \pi^{2}+1}{4 \log b}\right), \\
\phi_{n}(\xi) & =\sqrt{2} \xi^{-1 / 2} \sin (n \pi \log \xi),
\end{aligned}
$$

for $1 \leq \xi \leq b$, respectively. Moreover, $A(t)$ is the infinitesimal generator of a $C_{0}$-quasi-semigroup $R(t, s)$ given by

$$
R(t, s) x=\sum_{n=1}^{\infty}\left(\frac{t+s+1}{t+1}\right)^{\lambda_{n}}\left\langle x, \phi_{n}\right\rangle \phi_{n} .
$$

As the previous example, we assume that the system is controlled around the point $\xi_{c}$ and

$$
u(t, \xi)=u(t) \frac{1}{2 \epsilon} \chi_{\left[\xi_{c}-\epsilon, \xi_{c}+\epsilon\right]}(\xi)
$$

Condition (a) of Theorem 13 shows that system (78) is approximately controllable on $[0, \tau]$ if and only if, for all $n \geq$ 1 ,

$$
\beta(t) \int_{\xi_{c}-\epsilon}^{\xi_{c}+\epsilon} \xi^{-1 / 2} \sin (n \pi \log \xi) d \xi \neq 0 .
$$

Equation (83) confirms that the zeros of $b$ on interval $[0, \tau]$ affect the loss of approximate controllability.

Next, we locate the measurement $y(t)$ at the system output with the point measurement:

$$
y(t)=c(t) \frac{1}{\epsilon} \int_{b-\epsilon}^{b} x(t, \xi) d \xi
$$

where $c(t)$ is a continuous function. Condition (b) of Theorem 13 implies that the system is approximately observable on $[0, \tau]$ if and only if, for all $n \geq 1$,

$$
c(t) \int_{b-\epsilon}^{b} \xi^{-1 / 2} \sin (n \pi \log \xi) d \xi \neq 0,
$$

for some $\epsilon>0$. The Mean Value Theorem for integral implies that (85) is equivalent to

$$
\epsilon c(t) \xi_{n}^{-1 / 2} \sin \left(n \pi \log \xi_{n}\right) \neq 0,
$$

for some $\xi_{n} \in(b-\epsilon, b)$.

Remark 4. In this example we consider the nonautonomous regular Sturm-Liouville problem with the Dirichlet boundary condition. Actually, we can verify that all the results remain valid for which the problem has the Neumann boundary condition. Even, the nonautonomous singular Sturm-Liouville problems can be applied for the results. However, the periodic cases do not hold for the theory due to not simpleness of the related eigenvalues.

\section{Conflicts of Interest}

The authors declare that there are no conflicts of interest regarding the publication of this article.

\section{Acknowledgments}

The authors are grateful to Research Institution and Community Service of Universitas Sebelas Maret Surakarta for funding. This research is funded by Doctoral Dissertation Research Grant 2018 of Universitas Sebelas Maret Surakarta. 


\section{References}

[1] D. C. Venerus, "Analysis of finite length dynamics in Czochralski crystal growth," Journal of Crystal Growth, vol. 102, no. 3, pp. 376-386, 1990.

[2] A. Armaou and P. D. Christofides, "Robust control of parabolic PDE systems with time-dependent spatial domains," Automat$i c a$, vol. 37, no. 1, pp. 61-69, 2001.

[3] O. K. Dudko, A. M. Berezhkovskii, and G. H. Weiss, "Timedependent diffusion coefficients in periodic porous material," The Journal of Physical Chemistry B, vol. 109, no. 45, pp. 2129621299, 2005.

[4] J. R. Cannon, The One-Dimensional Heat Equations, Cambridge University Press, Cambridge, UK, 1984.

[5] D. Barcenas and H. Leiva, "Characterization of extremal controls for infinite dimensional time-varying systems," SIAM Journal on Control and Optimization, vol. 40, no. 2, pp. 333-347, 2001.

[6] A. Elharfi, H. Bounit, and S. Hadd, "Representation of infinitedimensional neutral non-autonomous control systems," Journal of Mathematical Analysis and Applications, vol. 323, no. 1, pp. 497-512, 2006.

[7] R. Schnaubelt, "Feedbacks for nonautonomous regular linear systems," SIAM Journal on Control and Optimization, vol. 41, no. 4, pp. 1141-1165, 2002.

[8] H. Bounit and S. Hadd, "Regular linear systems governed by neutral FDEs," Journal of Mathematical Analysis and Applications, vol. 320, no. 2, pp. 836-858, 2006.

[9] D. Barcenas, S.-N. Chow, H. Leiva, and A. Tineo Moya, "Skewproduct semi-flows and non-autonomous control systems," Journal of Mathematical Analysis and Applications, vol. 381, no. 1, pp. 247-262, 2011.

[10] J. Ng, S. Dubljevic, and I. Aksikas, "Aspects of controllability and observability for time-varying PDE systems," in Proceedings of the 2012 American Control Conference - ACC 2012, pp. 22202225, Montreal, QC, Canada, June 2012.

[11] X. Fu and Y. Zhang, "Exact null controllability of nonautonomous functional evolution systems with nonlocal conditions," Acta Mathematica Scientia, vol. 33, no. 3, pp. 747-757, 2013.

[12] S. Hadd, "An evolution equation approach to nonautonomous linear systems with state, input, and output delays," SIAM Journal on Control and Optimization, vol. 45, no. 1, pp. 246-272, 2006.

[13] H. Leiva and D. Barcenas, Quasi-semigroups, Evolution Equation and Controllability, Facultad De Ciencias Departemento De Matematica, Merida, 1991.

[14] D. Bárcenas, H. Leiva, and A. Tineo Moya, "The dual quasisemigroup and controllability of evolution equations," Journal of Mathematical Analysis and Applications, vol. 320, no. 2, pp. 691-702, 2006.

[15] Sutrima, Ch. R. Indrati, L. Aryati, and Mardiyana, "The fundamental properties of quasi-semigroups," Journal of Physics: Conference Series, vol. 855, no. 1, Article ID 012052, 9 pages, 2017.

[16] Sutrima, Ch. Rini Indrati, and Lina Aryati, "Stability of $C_{0}$-quasi semigroups in Banach spaces," Journal of Physics: Conference Series, vol. 943, Article ID 012021, 14 pages, 2017.

[17] P. Dolecki and D. L. Russell, "A General Theory of Observation and Control," SIAM Journal on Control and Optimization, vol. 15, no. 2, pp. 185-220, 1977.
[18] X. Zhao and G. Weiss, "Controllability and observability of a well-posed system coupled with a finite-dimensional system," IEEE Transactions on Automatic Control, vol. 56, no. 1, pp. 1-12, 2011.

[19] R. Rabah, G. Sklyar, and P. Barkhayev, "Exact null controllability, complete stabilizability and continuous final observability of neutral type systems," International Journal of Applied Mathematics and Computer Science, vol. 27, no. 3, pp. 489-499, 2017.

[20] R. F. Curtain and A. J. Pritchard, Infinite Dimensional Linear Systems Theory, vol. 8, Springer-Verlag, New York, NY, USA, 1978.

[21] R. F. Curtain and H. Zwart, "Linear Quadratic Optimal Control," in An Introduction to Infinite-Dimensional Linear Systems Theory, vol. 21 of Texts in Applied Mathematics, pp. 269-334, Springer, New York, NY, USA, 1995.

[22] H. C. Kuiper and H. J. Zwart, "Connection between the algebraic riccati equation and hamiltonian for riesz-spectral systems," Journal of Mathematical Systems, Estimation, and Control, vol. 6, no. 4, pp. 1-48, 1996.

[23] L. Paunonen, "Perturbation of strongly and polynomially stable Riesz-spectral operators," Systems \& Control Letters, vol. 60, no. 4, pp. 234-248, 2011.

[24] N. Dunford and J. T. Schwartz, Linear Operators, Part I: General Theory, Wiley (Interscience), New York, 1958.

[25] A. W. Naylor and G. R. Sell, Linear Operator Theory in Engineering and Science, Springer, New York, NY, USA, 1982.

[26] H. Sagan, Boundary and Eigenvalue Problems in Mathematical Physics, Wiley, New York, NY, USA, 1961.

[27] C. Delattre, D. Dochain, and J. Winkin, "Sturm-liouville systems are riesz-spectral systems," International Journal of Applied Mathematics and Computer Science, vol. 13, pp. 481-484, 2003. 


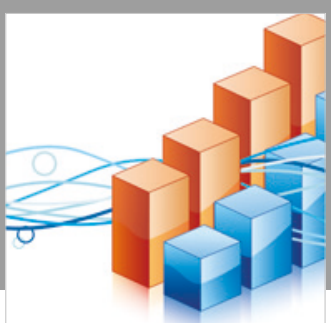

Advances in

Operations Research

\section{-n-m}
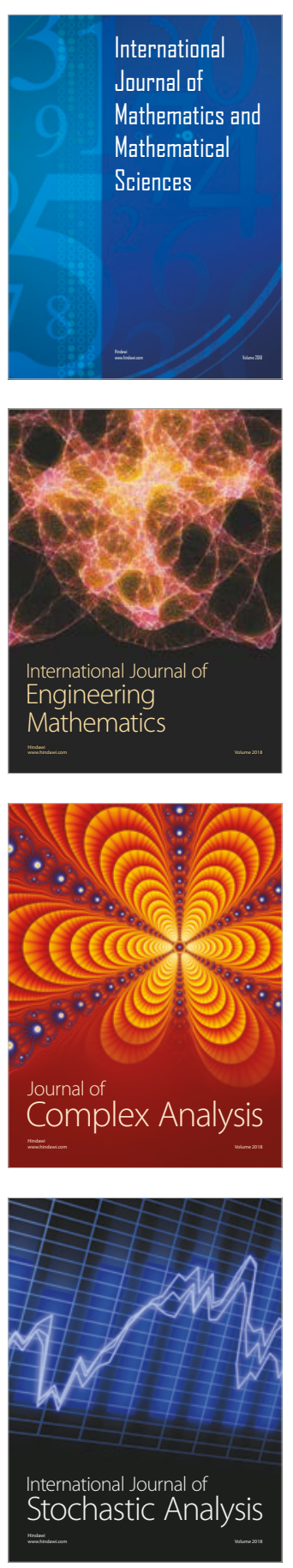
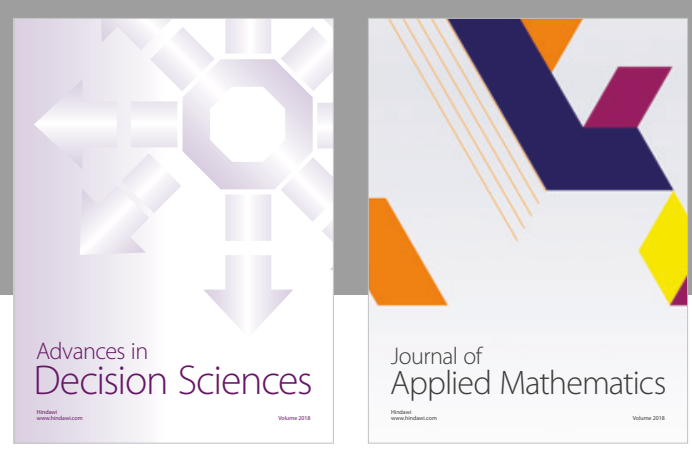

Journal of

Applied Mathematics
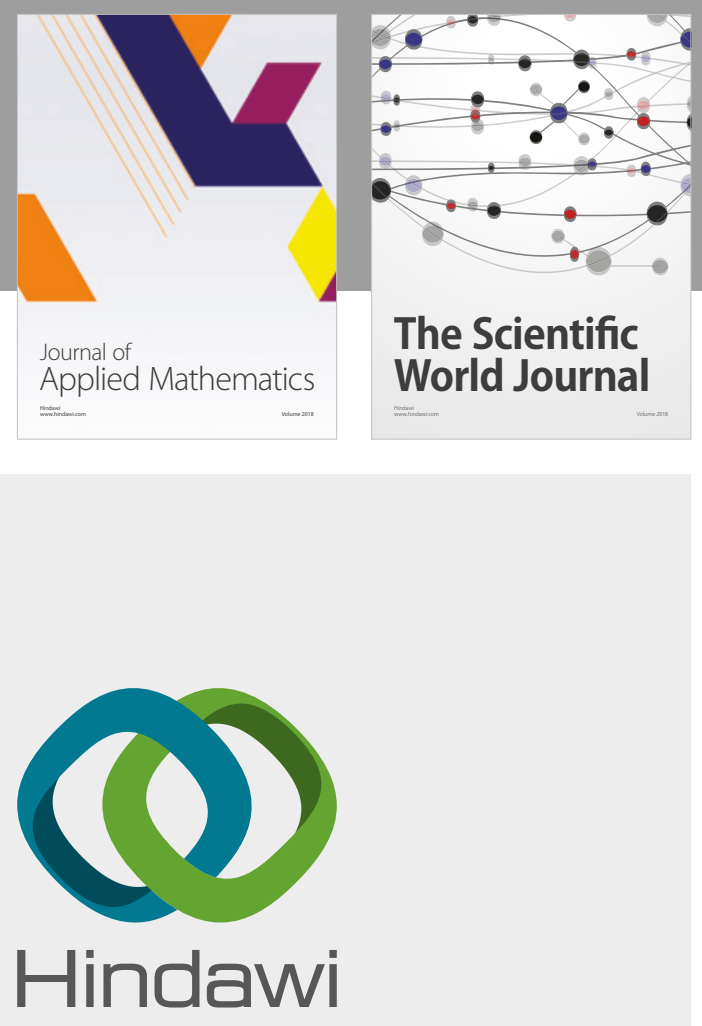

Submit your manuscripts at

www.hindawi.com

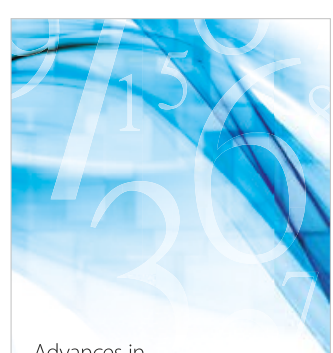

Advances in
Numerical Analysis
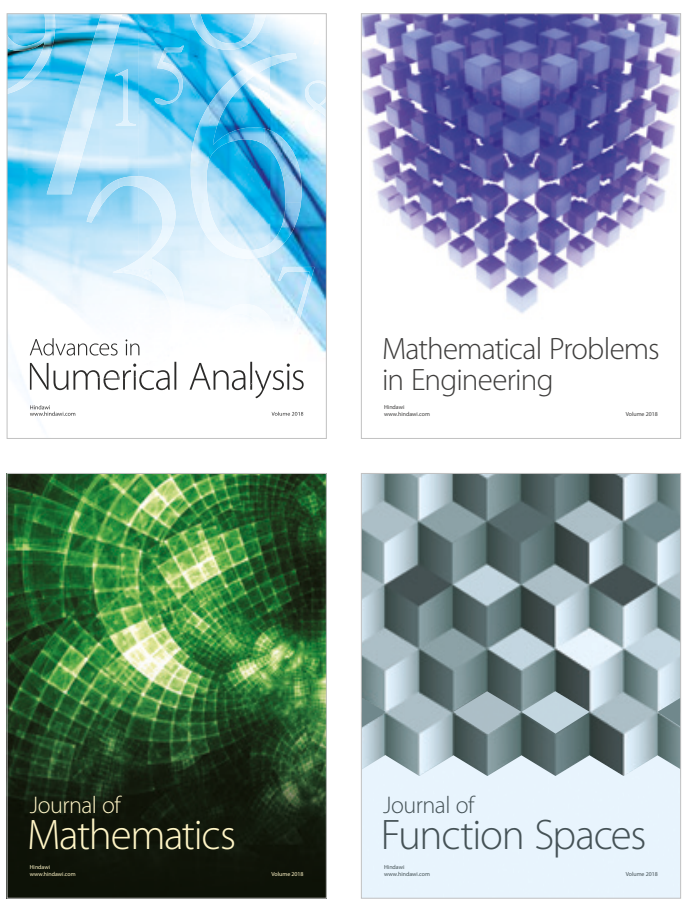

Mathematical Problems in Engineering

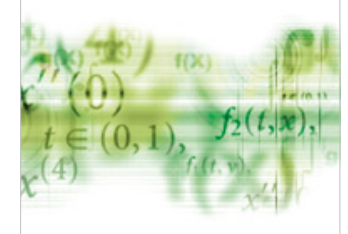

International Journal of

Differential Equations

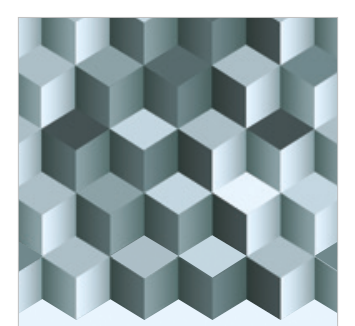

Journal of

Function Spaces

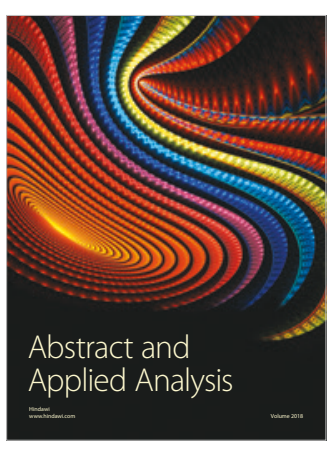

The Scientific

World Journal

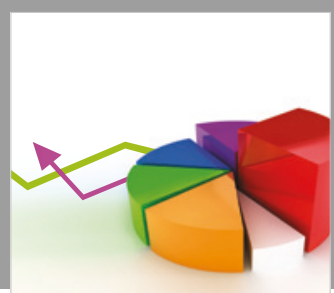

Journal of

Probability and Statistics
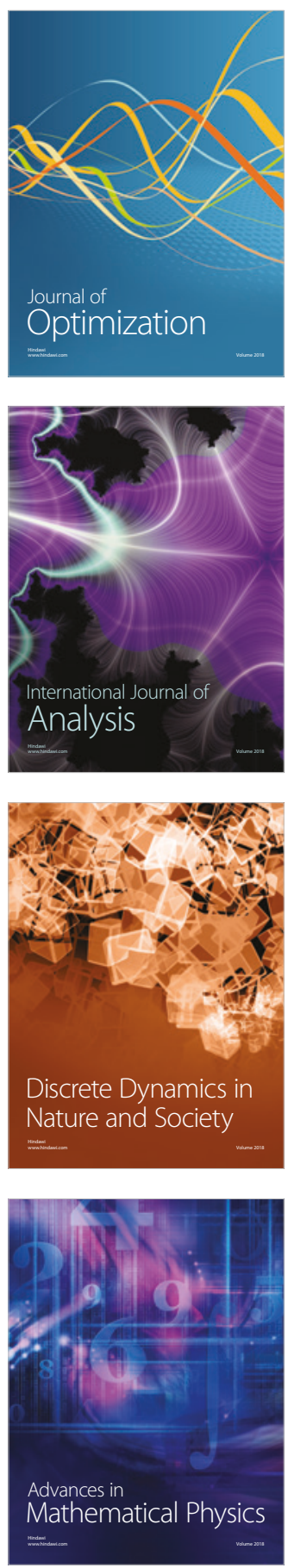